\title{
Modeling the operation of road pavement during the thawing of soil in the subgrade of highways
}

\author{
Aleksandr Isakov ${ }^{1, *}$, Denis Razuvaev ${ }^{1}$, Irina Gudkova ${ }^{1}$, and Michael Chakhlov ${ }^{1}$ \\ ${ }^{1}$ Siberian Transport University, Dusi Kovalchuk str., 191, Novosibirsk, 630049, Russia
}

\begin{abstract}
The solution for modeling the operation of road pavement during the thawing of soil in the subgrade of highways is proposed in this work. A model for layer-by-layer thawing of soil in the subgrade of highways was developed and illustrated, including the stage of modeling the change in the strength of the road structure during the thawing of soil, determining the dependence of the structural strength on the depth of soil thawing, thermophysical modeling of the process of freezing/thawing of soil in the subgrade using a software complex "Freeze-1" developed by the SSTU with determination of the dependence of the structural strength on time.
\end{abstract}

\section{Introduction}

Road pavement, like any construction structure, is designed taking into account the worst combination of environmental factors and the impact of adverse natural and climatic conditions that must be taken into account when assessing the strength of road pavement. When designing the road pavement on clay soil in the cold regions, an analysis of the change in its strength, depending on the seasonal change in the characteristics of the soil base, is needed. In such climatic conditions, the most unfavorable period for the operation of the road pavement is the period of spring thawing and consolidation of soil. Therefore, it is important to assess the change in strength of the entire road structure - pavement and its foundation, in the process of thawing the soil in the subgrade and its foundation.

In accordance with accepted standards, the assessment of strength of road pavement is carried out by measuring the deflection of its surface under a round stamp with a normative load. However, in this case, it is possible to assess the condition of road pavement after the fact, i.e. when the road is already built and nothing can be changed. The task of the designer is to predict the possible change in the strength and deformation characteristics of soil in the subgrade and to choose the design of road pavement that satisfies the worst conditions of soil.

To solve this problem, it is necessary to perform the modeling of operation of the road construction in the most unfavorable period of the year - during the thawing of soil in the subgrade of highways $[1,2]$. The purpose of this work is to build a model of road pavement deformation under a rigid stamp, taking into account the layer-by-layer thawing of soil in the subgrade of highways, which includes the following stages:

\footnotetext{
* Corresponding author: isakov@stu.ru
} 
- modeling of the change in the strength of a road structure with asphaltic concrete pavement during the thawing of soil;

- determination of the dependence of the change in the strength of road pavement on the depth of soil thawing;

- thermophysical modeling of the process of freezing/thawing of the subgrade with the help of the software complex "Freeze-1" [3,4] developed at the Siberian State Transport University and approved on the basis of the range of the Siberian State Automobile and Highway University [5].

\section{Model description}

The proposed model is based on the consideration of the change in the strength of a road structure in the spring, which occurs due to changes in the physical and mechanical characteristics of the thawing soil. The estimated zone is modeled by a multilayered medium consisting of constructive layers of road pavement, a layer of thawed soil of variable thickness hth, a layer of frozen soil, and a layer of soil that is not subject to seasonal changes in its aggregate state (see Figure 1).

The strength of a road pavement is assessed by the deflection value 1 [11] under the load of the round stamp, the diameter of which is equal to the diameter of the wheel imprint of the estimated car $(0.33 \mathrm{~m})$ with the load on the wheel of $50 \mathrm{kN}$.

The main and determining factor in modeling the change in the strength of a road structure is the distribution of total soil moisture in the subgrade at the beginning of its thawing.

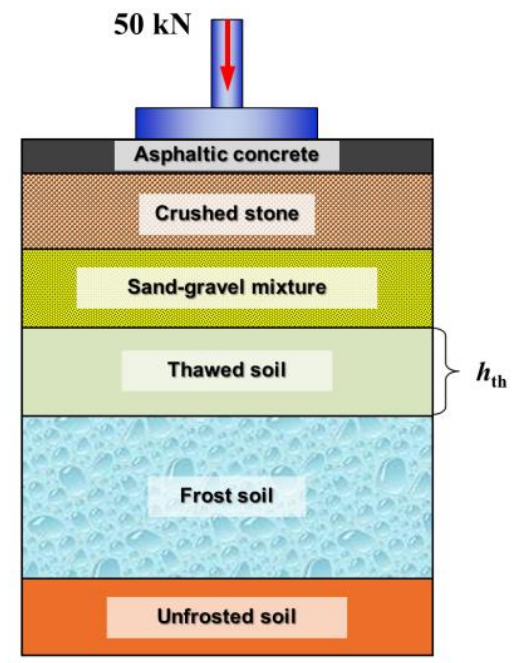

Fig. 1. The scheme of layers of road pavement and its foundation during the thawing of soil.

The scheme of the thawing of soil under the road pavement is shown in Fig. 2. By the beginning of the spring, this massif is represented by frozen soil (Fig. 2a). The actual distribution of relative moisture in depth is taken from the results of direct measurements before the beginning of the thawing period.

Further, a process of thawing is modeled layer-by-layer (with a step of 0.1-0.3 m) (Fig. 2b) until it's fully complete (Fig. 2c).

The sources of soil moistering in the subgrade are atmospheric precipitation, surface water, groundwater, and migration of water during the freezing of the subgrade $[6,7]$. 
a)

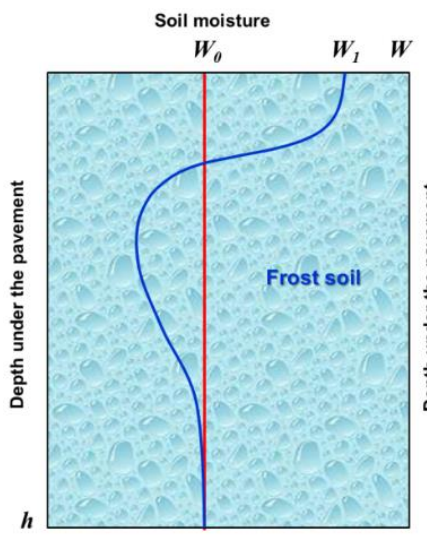

b)

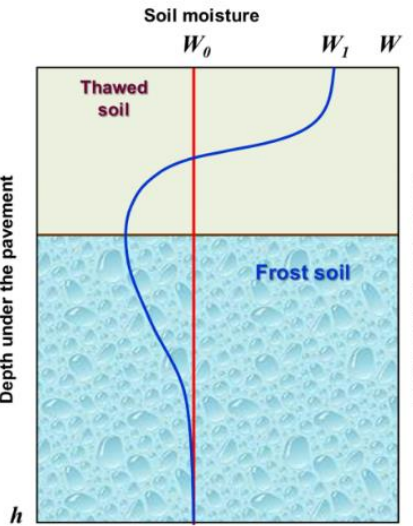

c)

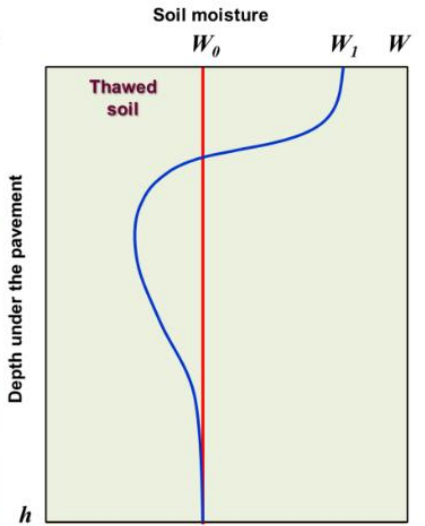

Fig. 2. The scheme of the thawing of soil under the road pavement, where: a) foundation in a frozen state; b) foundation in the process of thawing; c) thawed foundation.

The model alternately considers the phases of layer-by-layer thawing of soil, starting from a completely frozen state to a complete thawing (Fig. 3).

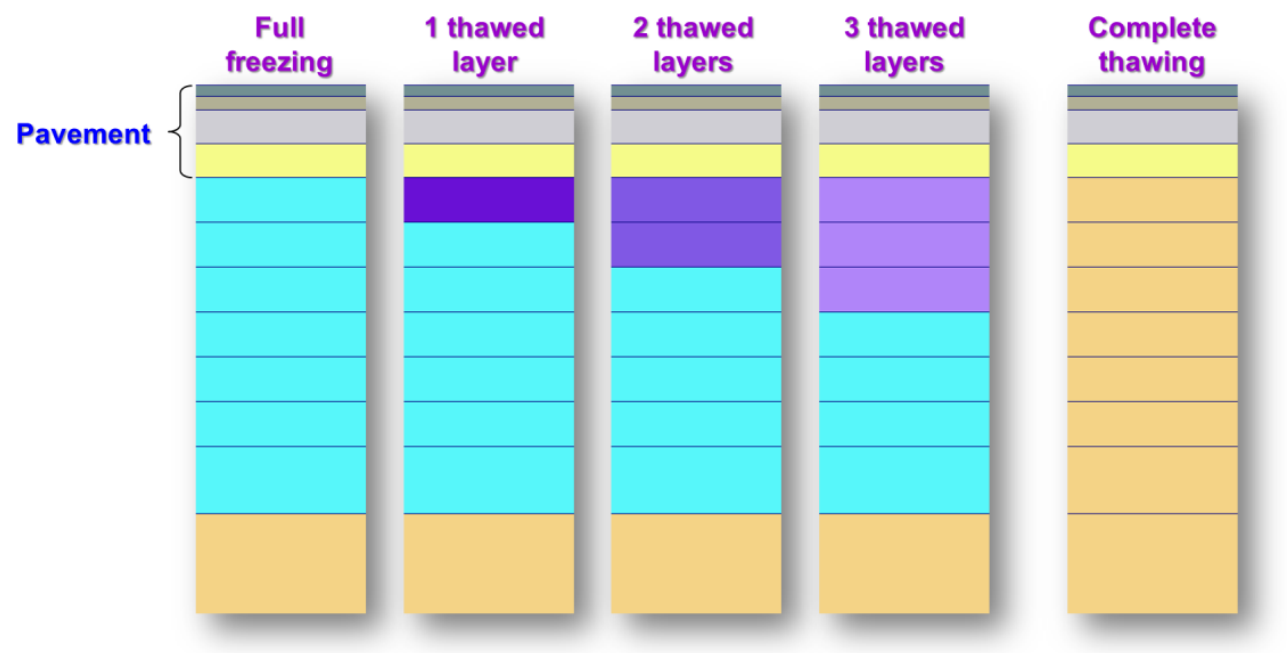

Fig. 3. Phases of layer-by-layer thawing of soil.

The main assumption here is the averaging of moisture in the depth of the layer of thawed soil under the road pavement using the given function of moisture depth distribution by the beginning of its thawing (Fig. 2).

In accordance with the accepted phases of soil thawing (Fig. 3), where moisture is a constant within each layer, point-to-point graph of the magnitude of pavement deflection is plotted, depending on the depth of the thawed soil layer (Fig. 4). According to the plotted graph, the depth of soil thawing in the subgrade $\left(h_{\text {crit }}\right)$ is established, corresponding to the maximum pavement deflection under the stamp $\left(l_{\max }\right)$, and, consequently, the maximum decrease in the strength of a road structure during the thawing and consolidation of soil. In Fig. 4 , the parameter $1_{\text {lim }}$ is the permissible magnitude of the pavement deflection, which ensures the normal operation of the subgrade. 


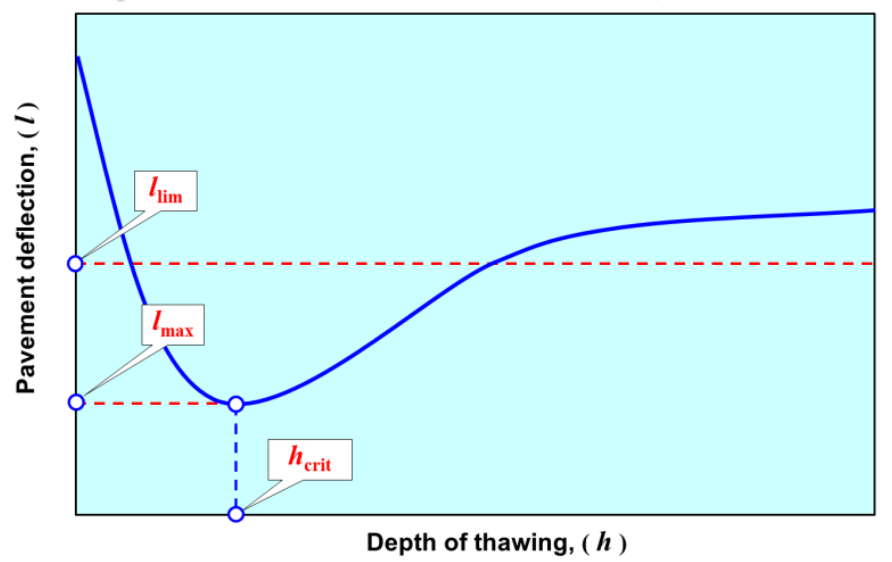

Fig. 4. Dependence of the pavement deflection on the depth of soil thawing.

Further, using the program "Freeze-1", the thawing of the road structure is calculated, taking into account the temperature data of the current year. At the same time, the time $\left(\mathrm{T}_{\text {crit }}\right)$ is set, corresponding to the maximum decrease in the strength of pavement during the thawing and consolidation of soil (Fig. 5).

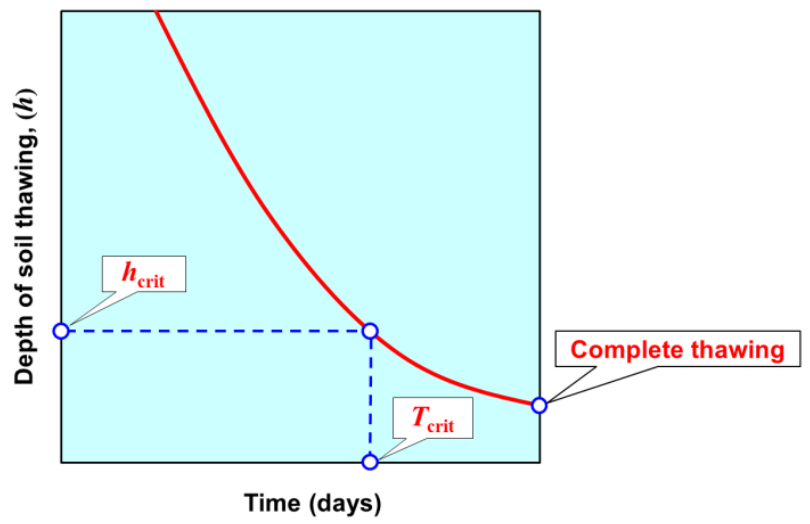

Fig. 5. Change in the depth of soil thawing under the pavement in time.

\section{An example of using the model}

The operation of the model is demonstrated on the example of the analysis of the state of the road structure on one of the sections of the "K-19r" highway in the Novosibirsk region. The selected section belongs to the IV category with heavy-duty pavement. The subgrade is represented by light silt loam from a solid to a high-plastic consistency (moisture at the plastic limit $\mathrm{W}_{\mathrm{p}}=0.20$, at the liquid limit $\mathrm{W}_{\mathrm{L}}=0.29$ ).

To plot the actual distribution curve for the relative moisture of the soil in the body of the subgrade before the thawing period, investigations were carried out that included fieldwork for the selection and packing of soil samples (Fig. 6) during the periods of the beginning of soil freezing (29.11.17) and their full freezing (09.03.18).

Then, under laboratory conditions, the physical characteristics of the selected soil samples were determined (natural moisture, moisture at the liquid and plastic limits). 


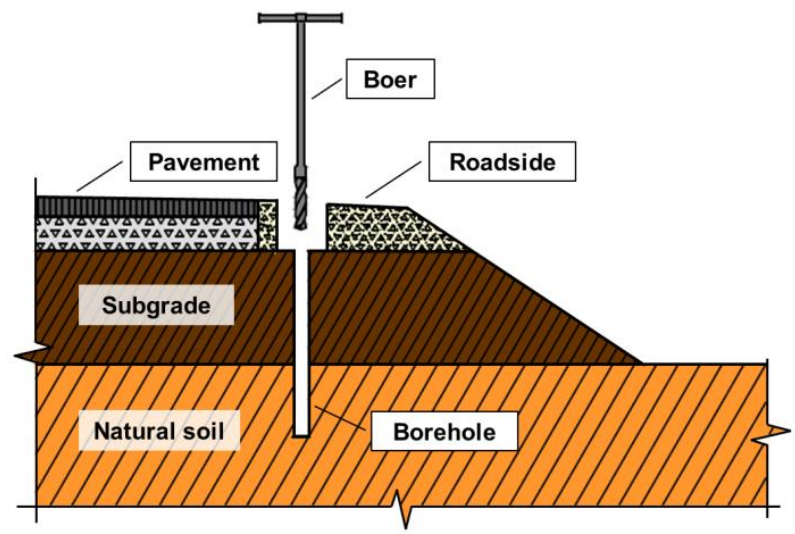

Fig. 6. The scheme of soil sampling at the object of the study using a hand boer $\mathrm{d}=40 \mathrm{~mm}$ in the unfrozen soil.

Based on the results of laboratory tests, graphs of the dependence of the relative moisture of the soil in the subgrade on its depth were plotted (Fig. 7).

\section{Relative moisture}

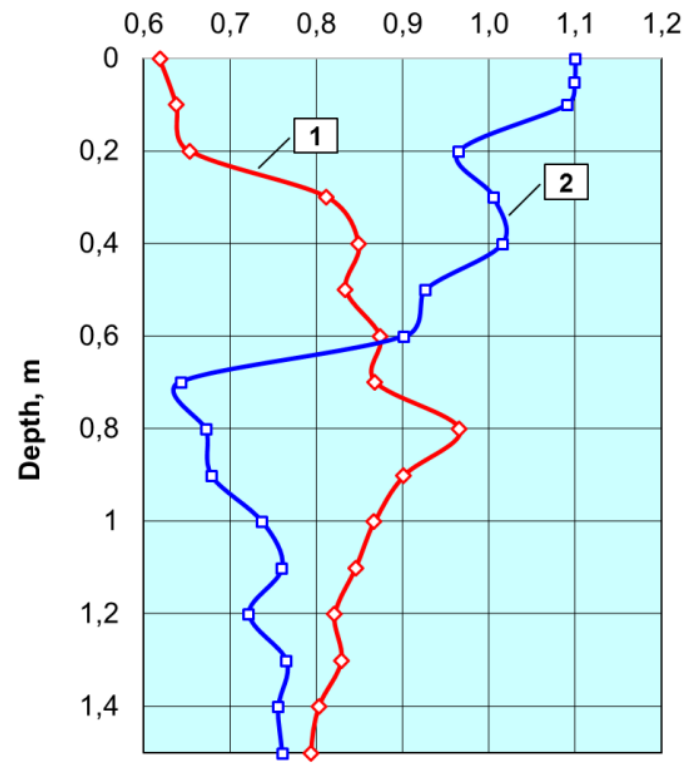

Fig. 7. Experimental dependencies of the relative moisture of soil (W/WL) on its depth under the pavement in the autumn and spring (1- pre-winter moisture (29.11.2017); 2- total moisture of frozen soil (09.03.2018)).

A comparison of the obtained graphs clearly demonstrates the effect of water migration from the underlying layers of soil to the zone of its freezing.

The analysis of the obtained results of the soil moisture change in depth and time confirmed the known mechanisms and ways of water migration in the working layer and the body of the subgrade, as well as the patterns of soil moisture distribution, namely:

- in the autumn, there is intensive moisture accumulation in the capping layer of the subgrade due to atmospheric precipitation; 
- in winter, there is a migration of loosely bound water to the soil freezing zone due to the film effect;

- the soil during thawing can become a fluid state, resulting in a sharp decrease in the strength of pavement.

The results of averaging the relative moisture values in depth of the thawed layer are shown in Fig.8.

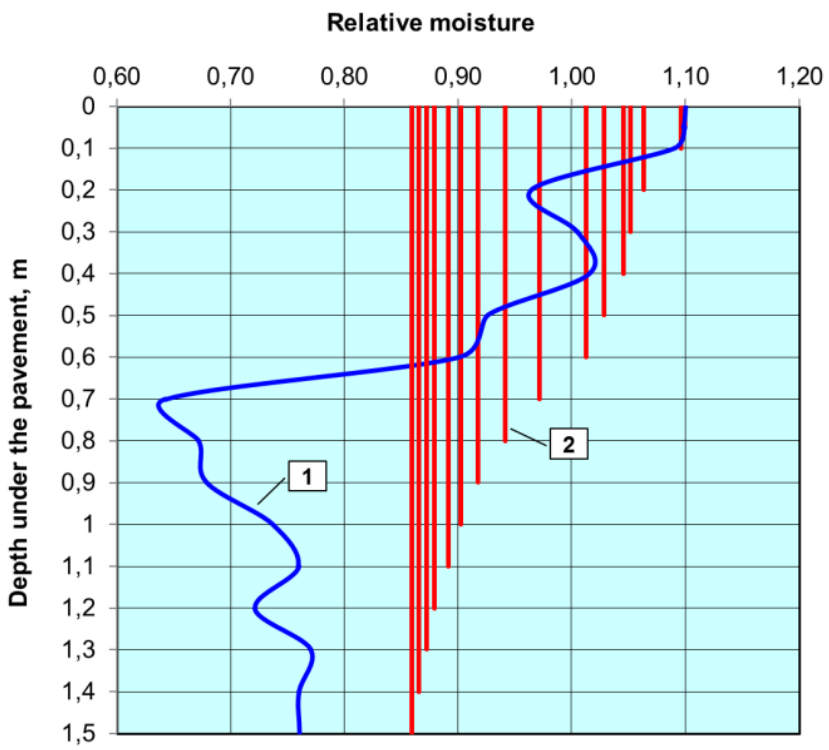

Fig. 8. The result of averaging the relative moisture of the thawing soil in the depth of the layers under the pavement (1-total relative moisture of frozen soil (09.03.2018); 2- averaged values of relative moisture in the entire depth of thawed soil layer).

The physical and mechanical characteristics of the thawed soil (Table 1) were taken according to Industry Road Codes and Standards (IRCS) 218.046-01. The elasticity modulus of soil in the solid-frozen and plastic-frozen state was taken as $500 \mathrm{MPa}$ and 200 $\mathrm{MPa}$, respectively. The construction of pavement on three different sections of the route was taken based on the results of cutting (Table 2). Characteristics of road materials are taken in accordance with IRCS 218.046-01. The load on the pavement structure was modeled by a rigid stamp (estimated imprint of the wheel) with a diameter of 0.33 meters, when the load on the car wheel is $50 \mathrm{kN}$ and an average pressure on the pavement is 0.6 MPa.

Table 1. Physical and mechanical characteristics of thawed soil.

\begin{tabular}{|c|c|c|c|c|}
\hline \multirow{2}{*}{$\begin{array}{l}\text { Thickness of } \\
\text { thawed layer } \\
\text { of soil, } m\end{array}$} & \multicolumn{4}{|c|}{ Average characteristics in the thawed layer } \\
\hline & $\begin{array}{c}\text { Relative } \\
\text { moisture } \mathrm{W}_{\text {rel }}\end{array}$ & $\begin{array}{c}\text { Elasticity } \\
\text { modulus E, MPa }\end{array}$ & $\begin{array}{c}\text { Specific } \\
\text { cohesion } \mathrm{C}, \mathrm{kPa}\end{array}$ & $\begin{array}{l}\text { Angle of internal } \\
\text { friction } \varphi \text {, degrees }\end{array}$ \\
\hline 0,2 & 1,06 & 14,4 & 18,2 & 11,8 \\
\hline 0,4 & 1,05 & 14,9 & 18,5 & 11,9 \\
\hline 0,6 & 1,01 & 17,1 & 20,0 & 12,5 \\
\hline 0,8 & 0,94 & 21,8 & 26,4 & 13,9 \\
\hline 1,0 & 0,90 & 25 & 27,5 & 14,8 \\
\hline 1,2 & 0,88 & 26,8 & 29,4 & 15,3 \\
\hline 1,5 & 0,86 & 28,7 & 31,1 & 15,8 \\
\hline
\end{tabular}


Table 2. Variants of pavement structure ("K-19r" highway).

\begin{tabular}{|c|c|c|c|}
\hline № & Layer material & $\begin{array}{c}\text { Thickness of } \\
\text { layer, } \mathrm{cm}\end{array}$ & $\begin{array}{c}\text { Elasticity modulus } \\
\text { E, MPa }\end{array}$ \\
\hline \multicolumn{4}{|c|}{ Structure №1 } \\
\hline 1 & Dense bituminous concrete $90 / 130$ & 6 & 2400 \\
\hline 2 & Porous bituminous concrete $60 / 90$ & 8 & 2000 \\
\hline 3 & Crushed stone-sand mixture & 20 & 450 \\
\hline 4 & Medium-grained sand & 10 & 120 \\
\hline \multicolumn{4}{|c|}{ Structure №2 } \\
\hline 1 & Dense bituminous concrete $90 / 130$ & 5 & 2400 \\
\hline 2 & Porous bituminous concrete $60 / 90$ & 6 & 2000 \\
\hline 3 & Crushed stone-sand mixture & 15 & 260 \\
\hline 4 & Medium-grained sand & 15 & 120 \\
\hline \multicolumn{4}{|c|}{ Structure №3 } \\
\hline 1 & Dense bituminous concrete $90 / 130$ & 5 & 2400 \\
\hline 2 & Porous bituminous concrete $60 / 90$ & 4 & 2000 \\
\hline 3 & Crushed stone-sand mixture & 10 & 260 \\
\hline 4 & Grit sand & 20 & 120 \\
\hline
\end{tabular}

Calculation of the structure deflection during soil thawing in the subgrade was carried out using the PLAXIS software complex. The deformed view of the road structure under the rigid stamp with the diameter of $0.33 \mathrm{~m}$ and with the force of $50 \mathrm{kN}$ is shown in Fig. 9.

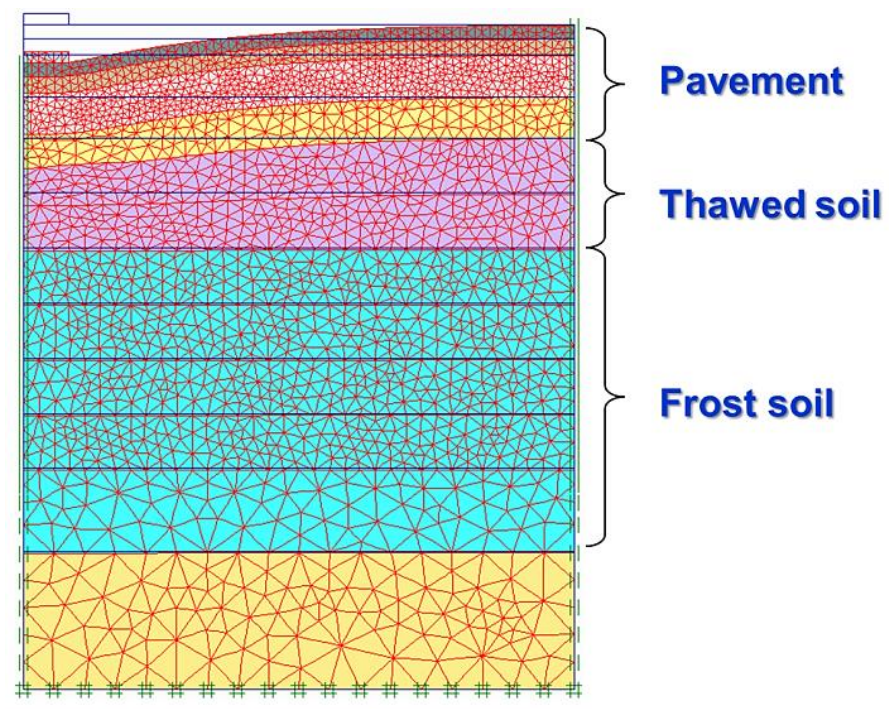

Fig. 9. The deformed view of the road structure under the stamp.

Based on the results of calculation, the graphs of dependence of the pavement deflection under the stamp on the depth of thawing are plotted for various pavement structures presented in Table. 2 (Fig. 10). 


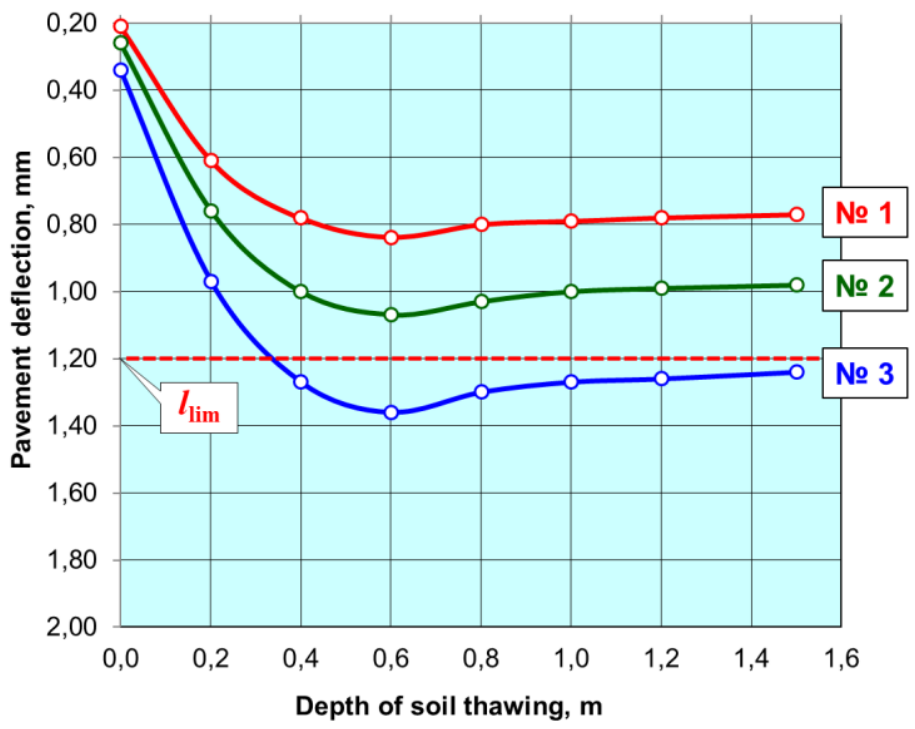

Fig. 10. Estimated dependencies of the pavement deflection from the depth of soil thawing for various pavement structures (Table 2).

As follows from the graphs above, only the pavement structures №1 and №2 meet the condition of the required strength $(1=1 \mathrm{lim})$ during the entire period of soil thawing. As for the structure № 3, it does not meet the requirements of the required strength during the entire period of operation, including the thawed state of the soil. For all pavement structures, the minimum strength is achieved with the same depth of soil thawing, which is equal to $0.6 \mathrm{~m}$. To assess the period at which the road structure of all three types (Table 2) reaches the minimum strength in the process of thawing of soil in the subgrade, a calculation of the process of seasonal freezing/thawing of the road structure is done using the "Freeze-1" software complex (Figure 11).

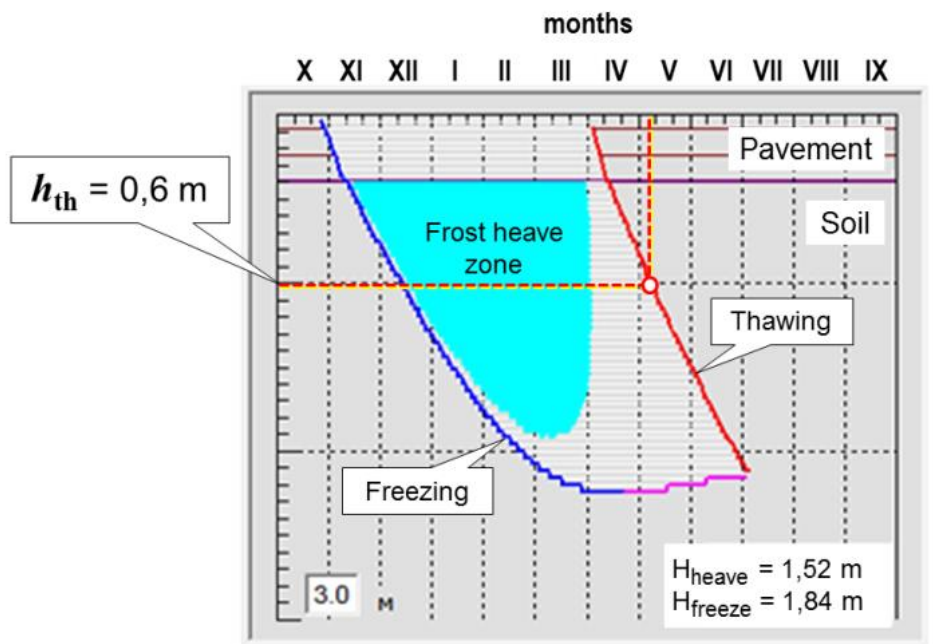

Fig. 11. Calculation of freezing/thawing of the road structure for the climatic conditions of Novosibirsk. 
As it follows from the graph of freezing/thawing of the subgrade shown in Fig. 11, the depth of the soil thawing $\left(\mathrm{h}_{\mathrm{th}}=0.6 \mathrm{~m}\right)$ critical for all three structures is reached in the first decade of May. This calculation was made in accordance with the average annual values of the average monthly air temperatures in the region of location of the investigated section of the highway. For specification of the specified period, it is necessary to set the average monthly temperature values corresponding to the specific year of pavement strength analysis.

\section{Conclusion}

The paper presents an algorithm for analyzing the strength of pavement in the process of soil thawing in the subgrade of highways, based on the discretization of this process layer-by-layer modeling of soil thawing. The proposed model is illustrated by the example of analysis of operation of three variants of pavement laid on one of the sections of the " $\mathrm{K}$ $19 \mathrm{r}$ " highway in the Novosibirsk region.

The results of the analysis showed that only two out of three variants of the road structure satisfy the strength condition (according to the deflection criterion) during the most unfavorable period of the year - seasonal thawing of soil. The depth of thawing, at which the strength of all three variants of the road structure assumes minimum values, turned out to be $0.6 \mathrm{~m}$. Based on the results of thermophysical modeling, the most unfavorable period for the highway operation on the considered section of the "K-19r" route was determined - the first decade of May.

The model described in this paper can be useful not only for analyzing the change in the strength of road pavement in the seasonal thawing of soil in the subgrade, but also in determining the terms for gentle operation of highways located in the cold regions.

\section{References}

1. S.V. Efimenko, Selected reports of the 60th University Scientific and Technical Conference of Students and Young Scientists, 129-133 (2015)

2. S.A. Kritskii, Selected reports of the II International Scientific Conference of Students and Young Scientists YST-2015, 313-318 (2016)

3. A.L. Isakov, Kim Hyun Chol, Proc.s of the VIII Scientific Conf., 137-142 (2011)

4. A.L. Isakov, Kim Hyun Chol, Transport of the Urals 2(33), 121-124 (2012)

5. E.V. Ivanov, A.L. Isakov, V.V. Sirotyuk, Bulletin of the Siberian State Automobile and Highway Academy 3, 71-76 (2013)

6. V.N. Efimenko, Sources of moistering of the subgrade of highways in the areas of deep freezing of soil. Investigation of transport facilities in Siberia (Publishing house of Tomsk University, Tomsk, 1987)

7. V.N. Efimenko, Water-thermal regime of the subgrade of highways with deep freezing of soil (On the example of the South-East of Western Siberia), thesis (Moscow, 1978)

8. Federal Law № 257-FZ of November 8, 2007 (as amended on 07.02.2017)

9. Order of the Ministry of Transport of the Russian Federation of August 12, 2011 N211

10. Methodical recommendations for determining permissible axle loads of vehicles in the spring on the basis of the results of diagnostics of public roads of federal significance: Road industrial methodical document 218.6.002-2010 (Informavtodor, Moscow, 2011)

11. Assessment of the strength of non-rigid pavements: Industry Road Codes and Standards 218.1.052-2002 (Informavodor, Moscow, 2003) 